\title{
Norois
}

Environnement, aménagement, société

$231 \mid 2014$

Les modes d'habiter à l'épreuve de la durabilité

\section{Les altermobilités : une mise en pratique des valeurs écologiques?}

Are altermobilities an application of ecological values?

\section{Stéphanie Vincent-Geslin}

\section{OpenEdition}

1 Journals

\section{Édition électronique}

URL : https://journals.openedition.org/norois/5112

DOI : 10.4000/norois. 5112

ISBN : 978-2-7535-3599-2

ISSN : $1760-8546$

\section{Éditeur}

Presses universitaires de Rennes

\section{Édition imprimée}

Date de publication : 30 septembre 2014

Pagination : 113-124

ISBN : 978-2-7535-3465-0

ISSN : 0029-182X

\section{Référence électronique}

Stéphanie Vincent-Geslin, «Les altermobilités : une mise en pratique des valeurs écologiques ? », Norois [En ligne], 231 | 2014, mis en ligne le 30 juin 2016, consulté le 14 janvier 2022. URL : http:// journals.openedition.org/norois/5112 ; DOI : https://doi.org/10.4000/norois.5112

\section{(c) Tous droits réservés}




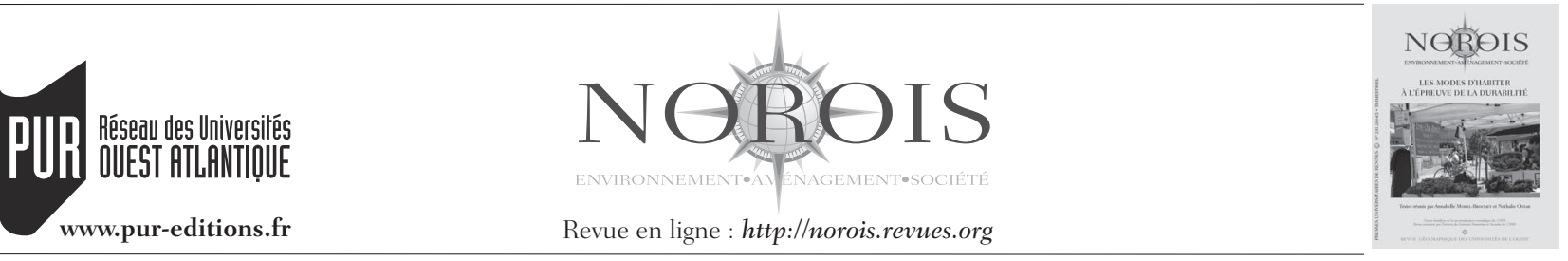

\title{
Les altermobilités : une mise en pratique des valeurs écologiques?
}

\author{
Are Altermobilities an Application of Ecological Values?
}

Stéphanie Vincent-GesLin

Laboratoire d'Économie des Transports (LET), ENTPE, Vaulx-en-Velin - chercheure associée au Laboratoire de Sociologie Urbaine (LASUR), EPFL, Lausanne, Suisse. (stephanie.vincent@entpe.fr)

Résumé : Au travers d'une réflexion sur les mobilités durables, appelées altermobilités, cette contribution questionne l'hypothèse d'une détermination des pratiques par les valeurs écologiques. Une réflexion théorique sur les valeurs en sociologie permet de remettre en question le caractère mécanique, normatif et unidirectionnel de l'influence des valeurs sur les pratiques. En effet, une multiplicité de valeurs peut être associée à une même pratique. Du reste, il apparaît que l'adoption des altermobilités s'explique bien plus par l'ordre des faits, sous l'influence des contraintes et des opportunités que par la présence des valeurs. Enfin, si la relation est majoritairement pensée dans le sens de l'influence des valeurs sur les pratiques, cette dernière peut également se renverser pour rendre compte de la manière dont la pratique même des altermobilités contribue à façonner des valeurs écologiques qui justifient et légitiment ces pratiques.

\begin{abstract}
This article questions if ecological values determine sustainable practices, through a reflection on sustainable mobilities, called altermobilities. A theoretical approach of the concept of values in sociology helps us questioning the automated, normative and unidirectional way of thinking the influence of values on practices. Thus, many values could be associated with the same practice. Moreover, processes of altermobilities are better explained by the order of facts, under the influence of constraints and opportunities, than by values. Finally, if the relationship is traditionally thought through the influence of values on practices, this could be reversed as altermobilities also contribute to the construction of ecological values, justifying and legitimating such altermobiliste practices.
\end{abstract}

Mots clés : sociologie - écologie - altermobilité - pratiques spatiales et sociales

Keywords: sociology - écology - altermobilitie - social and spatial practices

Depuis le début des années 2000, la question de la préservation de l'environnement a pris de plus en plus de place sur la scène médiatique mais aussi dans l'action publique. En outre, les grandes enquêtes d'opinion montrent une adhésion très forte des Français aux valeurs écologiques. Ainsi, les travaux de Jean-Pierre Bozonnet mettent en avant l'idée que « le grand récit écologiste qui a émergé dans les années 70 en Occident et qui correspond à une transformation majeure de la conception que les sociétés se font de leur rapport à la nature est désormais bien installé dans les esprits en France » (Bozonnet, 2009). Ce grand discours écologiste se caractérise en premier lieu par le passage d'un dis- 
cours anthropocentré à un discours écocentré qui ne place plus l'homme mais la nature au cœur du grand récit post-industriel (Bozonnet, 2009; Dunlap et al., 2000). En second lieu, il se caractérise par la conscience de la fragilité de l'équilibre naturel et des limites de l'économie face à cette fragilité (61\%) ainsi que par l'imminence d'une catastrophe écologique majeure si rien n'est fait pour limiter l'impact de l'homme sur la nature (87\%). Néanmoins, cette forte adhésion en valeurs peine à s'incarner dans des pratiques concrètes, écocitoyennes, militantes ou politiques et se heurte, en particulier, aux intérêts économiques. "Il existe donc un fossé entre un environnementalisme largement partagé et des pratiques assez éloignées des valeurs qu'il promeut » (Bozonnet, 2009). En matière de déplacements, le fossé entre pratiques et valeurs a été relevé par d'autres auteurs (Dobré et Juan, 2009), certains parlant même de "schizophrénie écologique » (La Branche, 2012).

Face à cela, de nombreux acteurs œuvrent pour transformer les comportements quotidiens trop consommateurs d'énergie et participant aux émissions de gaz à effet de serre : économies d'énergie dans le logement, meilleure isolation, ou encore usage de modes de déplacements moins énergivores et polluants que la voiture. Pour ce faire, ces acteurs, agences publiques, collectivités locales ou associations, souhaitent agir sur les comportements en promouvant un ensemble de «bonnes pratiques ». Ces bonnes pratiques, que ce soit dans le logement ou les déplacements, se réfèrent à un horizon moral - le bien commun - qui s'incarne dans la prise de conscience d'une urgence à préserver notre environnement. Dit autrement, ces acteurs ambitionnent de transformer les pratiques des citoyens en développant leur « conscience environnementale », donc en se référant à un idéal d'écologie et de préservation de l'environnement. Démarches d'éco-citoyenneté, éducation à l'environnement, recherche d'une prise de conscience environnementale individuelle et collective participent de ce mouvement. Ce faisant, ces acteurs stipulent un lien de causalité entre le développement de la conscience environnementale des citoyens et leurs comportements quotidiens, soit entre la référence et l'adhésion à un idéal de préservation de l'environnement et des pratiques quotidiennes durables. Plus précisément, pour la problématique spécifique des déplacements quotidiens, ce lien suppose que si les citoyens ont conscience que leurs déplacements en voiture vont à l'encontre de la préservation de l'environnement, ils abandonneront la voiture pour utiliser des modes de déplacements plus durables.

À partir de ce constat, cette contribution a pour objectif d'interroger la relation entre valeurs et pratiques dans l'adoption de comportements de déplacements durables - que nous appelons altermobilités. Les données empiriques utilisées ici proviennent d'une enquête qualitative par entretien réalisée en France en 2004 auprès de personnes dites « altermobilistes », c'est-à-dire d'usagers des altermobilités, au moins sur le trajet domicile-travail et ont constitué le corpus principal de notre recherche de doctorat (Vincent, 2008; Vincent-Geslin, 2010). Notre échantillon qualitatif se compose de cinquante personnes interrogées en France, dans les agglomérations de Lyon, Grenoble, Chambéry, Nîmes et Toulouse ${ }^{1}$. Pour constituer ce corpus, nous avons cherché à rencontrer la plus grande diversité possible de profils; des hommes et des femmes, actifs mais appartenant à toutes les classes sociales, aux situations familiales variées (en couple ou non, avec ou sans enfant), d'âges variés (de 26 à 62 ans) et aux situations résidentielles elles aussi multiples (centres urbains, périurbain voire zones rurales). La variété des villes étudiées, des contextes de localisation et de situations sociales et familiales permet ainsi de diversifier les contextes sociaux et spatiaux d'exercice de la mobilité quotidienne notamment en termes de densité et d'offre de transports ou encore de contraintes professionnelles et familiales - éléments qui contribuent à structurer les déplacements quotidiens.

Les « altermobilités » sont constituées de modes de déplacements alternatifs à la voiture, tels que les transports collectifs, le vélo ou encore le covoiturage. Ces modes de déplacements ont été retenus car ils participent d'une maîtrise de l'usage automobile et contribuent, à ce titre, à limiter l'impact des déplacements sur l'environnement. Les entretiens, menés avec la méthode du récit de vie ${ }^{2}$, rendent

1. Les personnes ont été rencontrées d'une part par l'intermédiaire de nos propres réseaux personnels et d'autre part par celle de structures de mobilité, telles que des associations de covoiturage ou de cyclistes. L'aide logistique et méthodologique que ces structures nous ont apporté dans le cadre de cette recherche a fortement influencé le choix des villes d'enquête.

2. Les récits de vie étaient centrés sur les modes de déplacements, depuis les souvenirs d'enfance jusqu'à la période actuelle. Ils ont donné lieu à des entretiens approfondis et longs, d'une durée comprise entre 1 h 30 et 3 heures. 
compte des usages et habitudes dans le temps long, sans négliger le sens qui leur est attribué. La méthode biographique permet alors de «prendre en charge théoriquement la question du passé incorporé, des expériences socialisatrices antérieures tout en évitant de négliger ou d'annuler le rôle du présent (de la situation)»(Lahire, 1998). Cette méthode très riche nous a ainsi permis de réfléchir aux processus de décision de l'altermobilité dans le temps long de la vie des personnes, en rendant visibles les héritages familiaux, les différentes expériences modales, des effets de génération et d'âge dans les usages de modes de déplacements ou encore l'influence des changements d'étape de cycle de vie sur ces derniers. Le moment du passage à l'altermobilité a été particulièrement travaillé durant les entretiens, ainsi que les représentations, opinions et valeurs des personnes sur les modes de déplacements et leurs usages avant, pendant mais aussi après le changement. Les diverses informations ainsi recueillies permettent alors une réflexion approfondie sur les liens entre comportements de mobilité durable et valeurs - en particulier les valeurs écologiques.

Les pratiques quotidiennes de déplacements et les choix qui sont opérés par les personnes ne sont bien sûr pas seulement liés aux valeurs auxquelles adhèrent ces personnes. Elles sont avant tout structurées par les espaces dans lesquels vivent et travaillent ces personnes, les contraintes temporelles auxquelles elles sont soumises tant par leur activité professionnelle que leur vie familiale, mais aussi les infrastructures à leur disposition et leurs propres compétences individuelles à les utiliser (Kaufmann et al., 2004; Kaufmann, 2000; Flamm, 2004). Tout en prenant en compte ces multiples dimensions sociales et spatiales qui construisent le choix des modes de déplacements, il s'agit d'opérer dans cette contribution un focus spécifique sur les liens entre valeurs écologiques - définies comme un idéal d'écologie et de préservation de l'environnement et pratiques de déplacements quotidiennes. Pour ce faire, nous reviendrons dans un premier temps sur un certain nombre de définitions et de caractéristiques des valeurs elles-mêmes. Ce cadrage théorique permettra ainsi de déconstruire le lien implicite entre valeurs écologiques et altermobilités, qui stipule que la présence de valeurs écologiques entrainerait des pratiques plus durables. En effet, ce lien implicite pose deux problèmes majeurs d'analyse en matière d'influence des valeurs sur les pratiques. En premier lieu, ce lien implicite pose comme présupposé que les pratiques de mobilité durable sont nécessairement associées à des valeurs écologiques, c'est-à-dire à un idéal de préservation de l'environnement. Or, un tel lien apparaît étroit et mécanique, voire normatif. Nous montrerons ainsi dans la seconde partie de cet article que les valeurs associées et associables aux altermobilités sont bien plus diverses que ce lien ne le laisse supposer. En second lieu, tout se passe comme si le développement des valeurs écologiques ou d'une conscience écologique, entrainait directement voire automatiquement davantage de comportements allant dans ce sens, c'est-à-dire de comportements durables tels que des usages alternatifs à la voiture. Or, un tel lien de causalité revient à nier d'une part le processus de sélection et d'activation différencié des valeurs dans l'action et d'autre part que les valeurs appartiennent à un autre ordre de phénomène que les actions. Ce faisant, ce lien implicite implique un déterminisme bien trop important des valeurs écologiques sur les processus de décision de la vie quotidienne. L'analyse des processus de décision de l'altermobilité présentée en troisième partie de l'article montre au contraire que le passage aux altermobilités se réalise principalement dans l'ordre des faits, sans intervention de l'ordre des valeurs sur le processus de décision. Enfin, si traditionnellement, à la suite de Weber, la sociologie considère que les valeurs orientent l'action, l'influence des premières sur cette dernière peut se questionner et s'inverser. Dans quelle mesure les pratiques altermobilistes contribuent-elles à l'élaboration de valeurs écologiques? Telle est l'ambition de notre dernière partie qui explorera ce renversement de perspective en puisant dans d'autres apports théoriques, en particulier dans l'interactionnisme et le constructivisme, qui permettent un nouvel éclairage sur notre problématique.

\section{DÉFINITION ET CARACTÉRISTIQUES DES VALEURS}

S'intéresser au rôle des valeurs dans les décisions humaines n'est pas chose aisée. En effet, s’il y a bien une chose sur laquelle s'accordent tous ceux qui travaillent sur les valeurs, c'est le flou et l'absence de conceptualisation de cette notion, que ce 
soit en anthropologie (Bonte et Izard, 2002), comme en sociologie. Ainsi Nathalie Heinich souligne que « le contraste est frappant entre l'omniprésence du thème des "valeurs » dans maints travaux se réclamant de la sociologie, et la pauvreté de ses conceptualisations»(Heinich, 2006). Qui plus est, la question du lien entre pratiques et valeurs trouve ses origines dans les travaux fondateurs de la discipline sociologique que sont les travaux de Max Weber ([1921] 1971) et qui posent l'hypothèse que les valeurs orientent l'action. Ainsi, déconstruire les liens entre pratiques de déplacements et valeurs écologiques implique de se positionner dans un débat beaucoup plus large de la sociologie, fortement marqué par l'influence wébérienne.

Travailler sur les valeurs en sciences sociales nécessite d'éviter deux écueils importants, d'une part celui de la philosophie morale et d'autre part celui du relativisme (Heinich, 2006). D'un côté, parler des valeurs d'un individu, d'un groupe ou d'une société ne consiste pas à dire, à la place de cet individu, de ce groupe ou de cette société, ce qui est bien et ce qui ne l'est pas, c'est-à-dire produire un discours normatif fortement éloigné de l'impératif de neutralité axiologique du sociologue. D'un autre côté, il ne s'agit pas non plus de tomber dans le relativisme et considérer que les valeurs ne seraient que des opinions car les valeurs possèdent, en tant qu'impératifs intériorisés, une puissance structurante des pratiques (Heinich, 2006). Les valeurs ne sont donc pas seulement des opinions individuelles et relatives, mais des idéaux plus profonds qui "structurent les représentations et les actions d'un individu »(Bréchon, 2000). Dans la tradition wébérienne, nous retiendrons pour notre propre positionnement que les valeurs sont reliées avec l'action à laquelle elles confèrent une orientation, un sens.

Néanmoins, si « les valeurs ont trait à des objectifs désirables qui motivent l'action » (Schwartz, 2006), les valeurs sont multiples et s'organisent en système. Elles font donc sens ensemble, les unes par rapport aux autres. D'après Schwartz, les actions humaines s'organisent à partir de 10 valeurs de base que l'on retrouve dans l'ensemble des sociétés humaines : l'autonomie, la stimulation, l'hédonisme, la réussite, le pouvoir, la sécurité, la conformité, la tradition, la bienveillance et l'universalisme qui entretiennent entre elles des rapports de compatibilité et d'antagonisme (Schwartz, 2006). Au sein de ce système, les valeurs sont classées selon un ordre d'importance et activées relativement les unes par rapport aux autres selon les contextes au moment d'agir (Schwartz, 2006). Les valeurs, multiples et parfois contradictoires, sont donc "vulnérables au contexte »(Heinich, 2006), c'est-à-dire qu'elles s'expriment différemment selon le contexte. En effet, nous pensons à la suite de ces auteurs que dans un contexte donné, les valeurs sont mobilisées tout comme les opinions, les représentations mais aussi les ressources et les contraintes pour aboutir à une action. De plus, selon les contextes, les valeurs d'une personne ne seront pas mobilisées et hiérarchisées de la même manière. Enfin, la vulnérabilité au contexte est d'autant plus importante que les valeurs interviennent avant, pendant et après l'action : «Les valeurs sont présentes trois fois : tout d'abord, elles existent avant la situation d'évaluation (c'est la dimension structurale, déterministe, de l'expérience commune); ensuite, elles s'élaborent dans la situation, en s'exerçant concrètement sur tel ou tel objet, avec une efficacité variable (c'est la dimension pragmatique et interactionniste); et enfin, elles se construisent après la situation d'évaluation, grâce à leur mise à l'épreuve au contact des objets et dans l'influence des contextes, de sorte qu'elles se modulent, s'affinent, s'affirment ou, au contraire, se périment, entraînant une réélaboration permanente du répertoire dont disposent les acteurs (c'est la dimension constructiviste et historiciste). » (Heinich, 2006)

Cette propriété de vulnérabilité au contexte a des implications directes en matière de méthodologie d'enquête pour saisir les valeurs et leur rôle dans l'action. Dans la littérature, un certain nombre de recherches procèdent de méthodes statistiques qui visent à mesurer les valeurs et leur importance relative au sein des sociétés, de certaines nations ou continents (Schwartz, 2006). Néanmoins, les valeurs appartiennent à un autre ordre de phénomène que les faits (Heinich, 2006; Bonte et Izard, 2002); de plus, elles ne sont pas toujours conscientisées par les individus au moment d'agir et sont, comme nous l'avons signalé ci-dessus, vulnérables au contexte. Pour toutes ces raisons, la méthode statistique ne permet pas d'appréhender véritablement « le degré d'explicitation ou de prise de conscience des valeurs en question, leur degré de pertinence pour les acteurs, ainsi que les modalités pragma- 
tiques de leur activation en situation vécue - toutes problématiques que permet en revanche de traiter l'approche compréhensive» (Heinich, 2006). C'est pourquoi, à la suite de Nathalie Heinich, nous nous situons dans cette contribution dans une démarche pragmatiste qui justifie pleinement la posture compréhensive et la méthode qualitative utilisées pour saisir les liens entre valeurs écologiques et pratiques altermobilistes et dépasser ainsi certaines apories de la méthode quantitative.

\section{UNE MÊME PRATIQUE, PLUSIEURS VALEURS}

Comme nous l'avons présenté ci-dessus, l'hypothèse selon laquelle la présence de valeurs écologiques chez les personnes entrainerait davantage de pratiques de déplacements plus durables revient à établir un lien trop étroit voire normatif entre valeurs écologiques et altermobilités. De plus, ce lien implicite constitue un exemple supplémentaire de la confusion relevée par Heinich entre « le principe de valorisation » - ici l'écologie - et « l'objet valorisé »-les altermobilités (Heinich, 2006). Pour le dire autrement, et en reprenant les dix valeurs de base mises au jour par Schwartz, comment savoir si les pratiques altermobilistes sont l'expression de valeurs telles que l'universalisme (dans laquelle Schwartz place l'écologie) qui appellent au dépassement des intérêts égoïstes (Schwartz, 2006) ou bien l'expression de l'hédonisme, de la stimulation ou encore de la tradition? En tant que telle, une pratique sociale ne peut être associée à telle ou telle valeur sans prendre en compte le sens que cette dernière revêt pour les personnes.

Ainsi, notre travail sur les altermobilités montre justement que ces pratiques de déplacements ne sont pas nécessairement jugées à l'aune de leur valeur écologique. En matière de déplacements, comme pour l'ensemble des pratiques quotidiennes, les pratiques sont évaluées selon différents étalons. Si la voiture est très souvent associée à un idéal d'autonomie, d'hédonisme ou encore à la stimulation à travers le plaisir de la conduite, elle n'est pas le seul mode de déplacements à renvoyer à de telles valorisations. Ainsi, l'usage du vélo peut être valorisé pour l'autonomie qu'il confère et ce dès le plus jeune âge :
«Le vélo, c'était un peu un rêve de me dire quand je serais étudiante, quand j'habiterais en ville, j'aurais le vélo! C'était vraiment une envie! Et vraiment, c'était un plaisir d'y aller en vélo, c'était pas l'envie de la voiture, se dire qu'on est indépendante! Non, c'était vraiment l'envie d'y aller à vélo, prendre l'air le matin... »(Sophie, 26 ans, Grenoble)

De même, les transports publics peuvent être utilisés dans une perspective ludique, répondant ainsi à des valeurs hédonistes ou de stimulation :

"De temps en temps même, avec un copain, on séchait les cours pour prendre les lignes de métro et aller le plus loin possible! Mais ça, faut peut-être pas le dire!! [Rires] »(Simon, 53 ans, Grenoble)

Les usages altermobilistes peuvent également être associés à la sécurité. Cette valeur sécurité se décline de deux manières différentes. Tout d'abord, elle entraine des usages automobiles notamment chez les femmes, en particulier lorsqu'elles doivent traverser certains espaces ou se déplacer la nuit. Dans ce cas, la voiture est considérée comme le mode de déplacement le plus sûr :

"J'ai 4 minutes de voiture à faire pour m'emmener à mon car! Je pourrais y aller à pied parce que à pied, il me faut 7 ou 8 minutes. Le problème, c'est que jusqu'à présent, il faisait nuit le matin mais maintenant, non! Il faisait nuit le matin, je traverse une petite zone vers chez moi qui est très lugubre et tout, donc je prenais la voiture pour m'arrêter vers un centre commercial. " (Sylvie, 54 ans, Grenoble)

«En voiture, je me sentirais plus en sécurité... Bon, à la période où ils parlaient beaucoup des vols aux feux rouges, dans les voitures, je fermais ma voiture à clé et puis c'est tout! Bon, c'est pas une sécurité pour tout, parce que si il m'arrive quelque chose, on ne peut pas ouvrir la porte! mais voyez, en voiture, je me sens complètement en sécurité... » (Jeanine, 55 ans, Chambéry)

Mais la valorisation de la sécurité peut également impliquer des déplacements en transports publics, afin d'éviter justement l'usage de la voiture qui est considéré dangereux. Ceci est particulièrement visible chez les jeunes femmes qui, ayant passé leur permis de conduire sous la pression de leur groupe familial, ont peur de conduire et mettent en place 
des stratégies d'évitement de la conduite (Vincent, 2008; Vincent-Geslin, 2010).

Enfin, les récits de vie mettent en lumière l'importance de la sobriété, de l'économie et de la lutte contre le gaspillage dans l'éducation de certains altermobilistes.

"L'attitude de mes parents était très fortement orientée "sobriété", non pas dans le sens de pénurie, mais d'attention à notre manière de vivre. Peu d'enfants à mon avis avaient un père qui s'amusait le dimanche soir à confectionner son propre Muesli au four. Ma mère m'expliquait qu'il ne fallait pas gâcher le papier, l'électricité, etc. Au total, j'en ai développé, il me semble, un certain penchant, même petit, pour "l'efficacité" et une aversion certaine pour le gâchis sous toutes ses formes, matérielles, mais aussi humaines. Enfin, je me demande si l'éducation religieuse que j'ai reçue, par ses valeurs de générosité, responsabilité, etc. n'a pas contribué à préparer le terrain, valeurs que j'ai ensuite étendues de la sphère sociale à une vision plus globale. » (Sébastien, 33 ans, Toulouse)

"Oui, y'a un truc qui me revient, j'ai eu une culture un peu écolo par mon père donc on avait le souci d'avoir des véhicules qui n'étaient pas gourmands donc le souci, je me rappelle, quand on est allé en Turquie, on avait dû étudier la consommation d'essence et on arrivait à 3 laux 100 à 3 dans une 2CV chargée! Donc on a eu quand même ce souci d'économies, toujours. Aussi sur la nourriture... mon père n'aimait pas qu'on achète, par exemple, des yaourts parfumés, comme on avait un jardin, il n'achetait jamais de produits chimiques pour le jardin, ni désherbant... mon père se soignait à l'homéopathie, je crois qu'il était relativement en avance sur son temps!»(Francis, 54 ans, Grenoble)

Ces valeurs peuvent s'apparenter à une forme d'écologie, et c'est d'ailleurs le lien que font ellesmêmes les personnes interrogées en les évoquant. Mais elles montrent également qu'une partie des altermobilités relève de pratiques traditionnelles et conformes à l'éducation familiale reçue, auquel cas les altermobilités apparaissent alors comme l'expression de valeurs de tradition et de conformité.

Enfin, les pratiques altermobilistes peuvent ne pas être du tout associées à des valeurs écologiques, remettant alors complètement en question un lien implicitement nécessaire entre pratiques altermobilistes et valeurs écologiques. En effet, certains altermobilistes rencontrés ont, durant l'entretien, nié de manière univoque et complète toute motivation ou valorisation environnementale dans leurs choix de déplacements :

"J'ai pas de citoyenneté par rapport à cette question. Souvent c'est un aspect que j'aborde pas. [...] Donc je n'ai pas des attitudes citoyennes par rapport à la vie écologique. Je ne me sens pas impliquée... (Catherine, 48 ans, Grenoble)

Ainsi, tous ces éléments montrent que l'attribution de certaines valeurs à certaines pratiques ne va pas de soi et relève avant tout du sens que leur attribuent les personnes qui les réalisent. Dit autrement, seuls les acteurs sont à même de donner le sens et les valeurs qui guident leurs actions, quand bien même ces dernières seraient multiples et diverses, d'où la nécessité de les interroger sur leurs pratiques concrètes et leurs décisions en situation. Quoique les valeurs écologiques soient fortes aujourd'hui, comment savoir si elles sont activées en situation concrète et de quelle manière? Comment s'opèrent la sélection et la hiérarchisation des valeurs dans les pratiques quotidiennes que sont les déplacements et en situation, c'est-à-dire en tenant compte des systèmes de contrainte dans lesquels se trouvent les individus? Pour cela, l'analyse des processus de décision apporte des clés de compréhension.

\section{DE LA Mise EN PRATIQUe DES VALEURS...}

Les recherches relatives aux valeurs s'accordent à dire que ces dernières ne s'expriment pas toujours de manière consciente chez les personnes. En effet, la sélection qui s'opère en situation entre différentes valeurs n'est pas toujours consciente (Schwartz, 2006; Heinich, 2006). De plus, les actions relèvent d'un autre ordre de phénomènes que celui des valeurs, l'ordre des faits, dans lequel agissent des ressources, des contraintes et des objectifs. Pour reprendre Leroi-Gourhan, cité par Chavanon et al. (2011), «dans les pratiques opératoires les plus courantes [...] de nombreuses actions sont faites dans un état de conscience crépusculaire " (LeroiGourhan, 1965). Ce constat a deux conséquences majeures en ce qui concerne notre travail d'élabora- 
tion des liens entre valeurs écologiques et pratiques altermobilistes : premièrement, il apparaît nécessaire de regarder les faits pour comprendre de quelle manière se prennent les décisions de l'altermobilité; deuxièmement, il apparaît également nécessaire de questionner de manière réflexive les personnes sur ces faits afin de rendre compte des valeurs mobilisées ou non dans ces décisions. C'est pourquoi cette seconde partie vise à analyser les processus d'adoption des altermobilités et à montrer la place qu'occupent dans ces processus les valeurs écologiques. La plupart des actions quotidiennes sont reproduites et répétées jour après jour sans que les personnes aient besoin d'y penser : elles deviennent alors des routines. Il en est ainsi des pratiques de déplacements comme de la plupart des pratiques quotidiennes : l'usage de modes de déplacements devient une habitude et ces habitudes entrainent la reproduction quasi-inconsciente des mêmes gestes qui fonctionnent comme "évidence intériorisée » (Kaufmann, 1997). De ce fait, les habitudes constituent un fort facteur d'inertie au changement (Kaufmann, 2000 ; Flamm, 2004). Face au poids inertiel de l'ordre des faits, quel rôle peuvent jouer les valeurs écologiques dans les processus de changement et à quels moments de ces processus interviennent-elles?

Les récits de vie se sont avérés particulièrement utiles pour mettre à jour les processus de décision de l'altermobilité. En effet, le changement modal est un processus complexe qui mobilise à la fois un contexte biographique favorable au changement, des dispositions à l'adoption d'un mode altermobiliste et enfin des éléments déclencheurs qui entraînent véritablement le passage à l'action. Tout d'abord, le changement modal s'insère dans un contexte biographique de remise en cause des routines. Cette remise en cause des routines peut prendre des formes extrêmement différentes, telle que la rupture biographique suite à un décès, le changement d'étape dans le cycle de vie (mariage, arrivée d'un enfant) ou encore une transformation affectant directement les trajets quotidiens (déménagement ou changement d'emploi notamment). Dans tous les cas, cet événement plus ou moins imprévisible affecte la trajectoire biographique de l'individu et remet en cause ses routines (Grossetti, 2006).

Ensuite, le changement d'habitudes modales ne peut se faire sans prédispositions à changer. Ces pré- dispositions peuvent également prendre des formes différentes : une «érosion de l'usage automobile » (Goodwin, 1985), l'attirance pour un autre mode, ou encore des valeurs écologiques. En effet, la présence d'une pensée réflexive sur les pratiques de déplacements chez une personne peut amener cette dernière à envisager et souhaiter l'usage d'autres modes de déplacements que la voiture afin d'être en cohérence avec ses valeurs environnementales. Ainsi, Anne (45 ans, Toulouse) se sentait « proche de toute la problématique liée à la protection de l'environnement » et a commencé à covoiturer quand elle a rencontré une autre personne partageant le même trajet et les mêmes horaires. De même, Léa (38 ans, Chambéry) baignait professionnellement dans un "état d'esprit de développement durable, transports durables » qui l'amenait à se dire «il faut que je fasse quelque chose». Ici, les valeurs environnementales interviennent donc avant le changement modal et fonctionnent comme prédispositions favorables au changement.

Néanmoins, pour que le changement ait lieu, celui-ci nécessite un élément déclencheur. Trois types de déclencheurs du changement ont pu être repérés : la contrainte, l'engagement et l'opportunité. Dans les processus déclenchés par la contrainte, le changement s'opère suite à la nécessité d'utiliser un autre mode de déplacement pour se rendre au travail (véhicule en panne, incapacité à conduire, etc.). Dans les processus opportunistes, le changement se trouve déclenché par la rencontre avec une alternative au déplacement automobile ou bien la mise en place d'une nouvelle offre. Il s'agit notamment des changements modaux que l'on observe lors de la mise en place de PDE (Plan de Déplacements d'Entreprise). Pour reprendre les exemples donnés plus haut, Anne (45 ans, Toulouse) a commencé à covoiturer suite à la rencontre avec son covoitureur tandis que Léa a commencé à utiliser le vélo pour se rendre au travail suite à l'ouverture d'une vélostation en gare de Chambéry. Enfin, dans les processus liés à l'engagement, ce sont bel et bien les valeurs environnementales qui se trouvent à l'origine du changement. Sous l'effet d'un processus de réduction de la dissonance cognitive (Festinger, 1957), certaines personnes fortement imprégnées de valeurs écocitoyennes qui, du reste, s'incarnent bien souvent dans des engagements associatifs et militants, 
changent de modes de déplacements pour mettre en accord leurs pratiques et leurs convictions. Changer de comportements est pourtant la modalité de réduction de la dissonance la plus difficile à mettre en œuvre. Mais, chez les altermobilistes militants, la dissonance est très forte du fait de l'importance subjective qu'ils accordent aux éléments cognitifs discordants (Poitou, 1974). En effet, l'engagement militant suppose l'implication de plusieurs personnes, qui deviennent autant d'observateurs de la dissonance. En renvoyant la perception de cette dissonance à l'individu qui la porte, ils la rendent davantage encore intolérable pour l'individu. De plus, l'exposition sociale de la dissonance constitue un risque pour la légitimité de tout le projet associatif ou militant. Ainsi, la résistance à la dissonance se trouve très faible, entraînant une plus forte mobilisation de ressources dans la réduction de cette dissonance, jusqu'à transformer les habitudes incohérentes avec leur manière de penser. Dans ce cas et uniquement dans celui-ci, les pratiques altermobilistes apparaissent comme le résultat de l'activation des valeurs écologiques. Ces processus de changement liés à l'engagement s'apparentent alors à l'action rationnelle en valeurs, selon la terminologie de Max Weber (Weber [1921] 1971).

Ainsi, les valeurs écologiques interviennent en amont des processus de changement comme dispositions à changer de mode de déplacements, ou bien comme déclencheur de ce changement sous la forme du militantisme. Néanmoins, ce sont bien les éléments pratiques, les contraintes et opportunités qui s'offrent aux personnes qui expliquent majoritairement les changements modaux observés. Les valeurs écologiques apparaissent alors, contrairement au lien implicite voire déterministe largement établi, ni nécessaires ni suffisantes au changement d'habitudes modales.

\section{... À LA MISE EN VALEUR DES PRATIQUES?}

Si nous avons jusqu'à présent exploré l'influence des valeurs sur les pratiques altermobilistes, il s'agit désormais de renverser la perspective afin de questionner le rôle de ces pratiques dans l'élaboration de valeurs écologiques. En effet, notre recherche met également en lumière le fait que les pratiques altermobilistes influencent la pensée réflexive des personnes et peut amener à développer des valeurs écologiques.

Certains altermobilistes, en particulier ceux pour lesquels la pratique alternative est ancienne, construisent progressivement au cours de leur « carrière » (Becker, 1985) d'altermobiliste une véritable idéologie de la pratique. Cet état de fait est particulièrement visible chez les altermobilistes cyclistes, dont la pratique est ancienne, et qui construisent et renforcent leur pratique tout au long de leur vie dans l'interaction avec d'autres personnes altermobilistes et militantes. Ces personnes qui adoptent des pratiques altermobilistes constantes au cours de leur vie - constance qui constitue d'ailleurs le quatrième processus amenant aux altermobilités (Vincent, 2008; Vincent-Geslin, 2010) - développent des habitudes de déplacements qui s'apparentent à des carrières au sens de Becker car on y retrouve les étapes amenant à une pratique déviante stable (Becker, 1985). En effet, les cyclistes militants ont construit leur pratique, mais aussi les représentations et justifications qui lui sont associées au fil du temps notamment dans l'interaction avec les autres pratiquants et militants cyclistes. On observe le même processus que dans les carrières déviantes : ce ne sont pas les motivations qui amènent à la pratique, mais bien la pratique et le contact avec le groupe qui construisent les manières de faire et de penser du cycliste (Becker, 1985). Dans leurs discours, ces personnes mettent en avant l'ancienneté et la continuité de leur pratique cycliste, dont les origines remonteraient à l'enfance et aux trajets scolaires. Les cyclistes militants insistent également sur leurs fortes convictions écologiques qui seraient au cœur voire à l'origine de leur démarche :

"C'est amusant, depuis qu'on s'est vu à Toulouse, je suis allé rendre visite à mes parents, qui m'ont montré un petit dessin que j'avais fait à l'âge de 7 ans. Au dos j'avais écrit qui j'étais et ce que j'aimais. J'avais notamment écrit "Je n'aime pas la ville, je n'aime pas la pollution" [sic]... Sans que mes parents m'aient parlé de respect de l'environnement...» (Sébastien, 33 ans, Toulouse)

Pourtant, la reconstitution des étapes de la carrière de ces cyclistes militants montre que ces valeurs relèvent davantage d'une idéologie formée dans la pratique que d'une motivation originelle à 
cette dernière. En effet, les différentes étapes de la « carrière », telle que décrite par Becker, se retrouvent effectivement chez les altermobilistes cyclistes militants, y compris celle de l'étiquetage, certains cyclistes passant auprès de leurs proches pour des « fous ». Au départ, pratique occasionnelle, voire adoptée à l'insu des parents, elle devient plus régulière et plus pérenne par la suite du fait du développement de motifs et intérêts. Sa stabilité apparaît également fortement liée aux interactions avec d'autres cyclistes puis à l'entrée dans un groupe cycliste organisé, tel qu'une association :

«Après, je suis allé à Londres, pendant 3 ans, où là, j'étais cycliste du début à la fin! Le métro, à Londres, de temps en temps, je le prenais mais c'est vraiment pas pratique! C'est super cher! Moi, j'avais récupéré un vélo correct et voilà... J'ai habité dans trois endroits différents. Au début, c'était tout près de là où je bossais, après, c'était très loin, à 13 km et après, c'était à peu près entre les deux, à $6 \mathrm{~km}$. J'ai toujours pris le vélo... [...] Et à l'époque, j'essayais d'être un peu plus militant! J'ai rejoint un groupe qui s'appelait London Cycling Campaign, l'équivalent de l'association pour la promotion du vélo à Londres et puis, je vivais avec des gens... À Londres, on est souvent en colocation, parce que les loyers sont tellement chers! Des gens qui étaient aussi très anti-bagnoles donc c'était convergence d'intérêt! [...] L'association générale était le London Cycling Campaign. J'étais notamment actif, de par mes activités professionnelles, dans le Cycling Public Affairs Group, le groupe chargé spécifiquement du lobbying pro-vélo auprès des autorités nationales. » (Sébastien, 33 ans, Toulouse)

Le partage d'un logement avec des personnes également cyclistes, l'appartenance à des milieux professionnels similaires ainsi que l'engagement militant dans l'association londonienne cycliste amènent ainsi Sébastien à renforcer son appartenance à une « sous-culture » cycliste, à en prendre et en apprendre non seulement les routines, les manières de faire mais aussi les façons de penser, les justifications, c'est-à-dire toute son «idéologie » (Becker, 1985). Ce partage de valeurs et de pratiques communes l'amène alors à une pratique "plus réfléchie et plus cohérente » (Becker, 1985) que nous retrouvons notamment dans ses justifications idéologiques. Ainsi, si le cycliste militant tendait à vou- loir expliquer l'ensemble de ses pratiques par des valeurs - y compris le domaine dans lequel il évolue professionnellement - cette rhétorique semble avoir été construite au travers même des interactions avec les autres cyclistes et de la sous-culture ainsi développée. «Ce ne sont pas les motivations déviantes qui conduisent au comportement déviant, mais, à l'inverse, c'est le comportement déviant qui produit, au fil du temps, sa motivation déviante » (Becker, 1985).

Ainsi, l'enracinement de pratiques altermobilistes au sortir de la jeunesse apparaissent, pour une part, davantage lié aux « contingences » (Becker, 2002) des rencontres et des appartenances sociales qui engagent les individus dans la voie du militantisme. Constituant une sorte de sous-culture modale, les cyclistes militants partagent ainsi leurs manières de faire et de penser et contribuent à créer une idéologie qui leur est propre. Des effets de socialisation et d'appartenance très forts, plus que les valeurs dont le rôle est souvent survalorisé par les enquêtés, se trouvent être à l'origine de l'enracinement de pratiques altermobilistes constantes, dès l'entrée dans la vie adulte. La pratique des altermobilités tout au long de la vie participe donc de la création d'une idéologie, fortement reliée à des valeurs écologiques et citoyennes. En effet, pour ces cyclistes, comme pour toute pratique déviante, la création de cette idéologie s'avère nécessaire pour affronter le regard stigmatisant des autres, justifier la pratique et ainsi la renforcer.

Plus généralement, l'ensemble des pratiques altermobilistes nécessite, pour perdurer dans le temps, une légitimation sociale car elles sont très largement encore considérées comme des pratiques étranges ou anormales en regard de la norme d'usage automobile (Vincent, 2008). Il est certainement utile de rappeler ici que le terrain qui constitue la base empirique de cet article a été réalisé en 2004, c'est-à-dire avant le lancement des vélos en libre-service, de l'engouement des villes françaises à l'égard du tramway et du développement de services d'autopartage et de covoiturage. Toutes ces initiatives ont largement contribué à valoriser d'autres usages de déplacements que la voiture particulière et à légitimer, par leur aspect innovant et leur présence même dans l'espace public, les altermobilités - pensons ici par exemple aux Velo'v, ainsi qu'aux plus récents systèmes de voiture en libre- 
service. Mais, dans les entretiens réalisés en 2004, ce mécanisme de légitimation était principalement permis par la reconnaissance par les proches des nouvelles pratiques comme des pratiques légitimes. Par analogie avec les processus de conversion religieuse, « ce qui compte vraiment, c'est être capable de continuer à la prendre au sérieux; de conserver le sens de sa plausibilité. » (Berger et Luckmann, 2006). C'est bien la pérennité des nouvelles pratiques altermobilistes qui est en jeu ici. Ainsi, les changements modaux qui interviennent dans le cadre de Plans de Déplacements sont facilement légitimés par l'existence de ce Plan même; les changements qui interviennent dans certaines agglomérations de résidence le sont également plus que dans d'autres ${ }^{3}$. Lorsque les proches ne légitimaient pas les nouvelles pratiques mais tendaient, au contraire, à les stigmatiser, les altermobilistes tendaient à chercher de nouvelles instances de légitimation auprès notamment d'autres usagers d'altermobilités. Pour cela, certains altermobilistes s'engageaient dans des pratiques militantes, notamment associatives, qui leur apportaient une forme de légitimation, de la même manière que la communauté religieuse légitime la conversion d'un individu (Berger et Luckman, 2006). Mais, en matière de légitimation, une grande partie des altermobilistes rencontrés se référaient aux valeurs écologiques pour justifier leurs pratiques.

Si l'ordre des faits, des ressources et des contraintes, préside au changement de modes de déplacements, les valeurs interviennent à titre de motivation générale ou de justification :

"Ça fait partie de mes motivations. Je pense que mon épouse et moi avons quand même une sensibilité particulière à l'environnement, la protection de l'environnement. J'ai le sentiment que c'est quelque chose qui nous préoccupe beaucoup plus que d'autres personnes et donc c'est vrai que ça participe de ce sentiment-là. Ça va pas jusqu'à aller à pied! Dans certains cas, vous pourriez me dire que je pourrais $y$ aller à pied ou prendre le bus... J'ai conscience, je sais de façon intellectuelle que prendre le bus est quand même un moyen plus écologique que prendre ma voiture pour aller au cinéma! Eh ben, je ne vais

3. Elles sont notamment facilitées par l'existence de politiques urbaines, d'infrastructures, d'initiatives favorables aux altermobilités ainsi que de campagnes de promotion des altermobilités. pas jusque là! Ma conscience a des limites... C'est pas ma motivation première pour prendre mon vélo... » (Marcel, 46 ans, Nîmes)

Pour Marcel, comme pour bien d'autres altermobilistes rencontrés, ce sont avant tout les contraintes et les opportunités qui ont guidé les changements modaux, même si l'environnement est une valeur qui fait sens pour eux. Les valeurs environnementales interviennent alors a posteriori du changement modal, comme une justification aux nouvelles habitudes de déplacements. Le fait que les nouvelles habitudes modales soient positives en regard de l'idéal de préservation de l'environnement fonctionne comme un avantage supplémentaire voire inattendu au changement de pratiques :

«Bon, y'a aussi un peu mettre en accord mes actes avec ce que je pense au niveau de l'environnement... [...] Y'a aussi le côté environnement, je me dis que c'est peut-être pas mal de prendre le train, plutôt que la voiture! »(Patrice, 41 ans, Chambéry)

C'est le principe d'" une pierre deux coups » repéré par d'autres auteurs en matière d'environnement (Chavanon et al., 2011). Néanmoins, en invoquant l'importance de la sauvegarde de l'environnement, les altermobilistes se réfèrent à des valeurs sociales admises par tous ce qui limite l'anormalité de leurs nouvelles pratiques. Les valeurs écologiques interviennent alors ici non pas avant ou pendant le changement mais bien après. Elles participent alors de la pérennité des nouvelles pratiques de déplacements comme instances de légitimation de ces dernières. Néanmoins, même a posteriori, les valeurs environnementales peuvent ne pas intervenir dans la pratique des altermobilités, les raisons pratiques suffisant à expliquer et justifier le choix des altermobilistes.

Dans notre corpus d'entretiens, le rôle des valeurs environnementales comme justification dans la pratique était fortement répandu. Même si nous ne pouvons pas l'affirmer pour les raisons méthodologiques inhérentes à toute enquête qualitative, nous posons l'hypothèse que les valeurs environnementales interviennent principalement a posteriori des changements modaux comme justification et légitimation de ces changements et jouent finalement un rôle relativement limité dans les processus de changement eux-mêmes. Ainsi, l'hypothèse selon 
laquelle les valeurs environnementales interviennent plutôt a posteriori du changement de mode de déplacement et se construisent dans la pratique constitue un renversement complet de perspective face aux idées communément admises. Néanmoins, cette hypothèse semble corroborée par certaines initiatives politiques récentes qui constituent des stratégies de légitimation de comportements de déplacements durables. Rappelons par exemple que l'autopartage, aujourd'hui promu comme modalité de déplacement alternative à la voiture personnelle, est à l'origine né d'initiatives collectives privées. Ou encore que l'aménagement d'aires de covoiturage ne fait que légitimer des pratiques informelles déjà existantes. De telles stratégies de légitimation contribuent pourtant à alimenter un cercle vertueux en matière de mobilité durable en affaiblissant progressivement la norme du «tout voiture » et en rendant toujours plus familiers - devrait-on dire normaux? - les modes et usages de déplacements alternatifs à la voiture.

\section{Conclusion}

A partir du constat d'une schizophrénie écologique, c'est-à-dire d'une dissonance, fortement visible aujourd'hui en Europe et en particulier en France, entre l'adhésion aux valeurs écologiques et des pratiques non écologiques, cette contribution a plus particulièrement exploré les liens entre valeurs écologiques et altermobilités. Nos résultats montrent que les valeurs n'interviennent pas de manière déterministe ni déterminée sur l'adoption de pratiques de déplacements alternatives à la voiture. En effet, valeurs et pratiques appartiennent bien à deux ordres de phénomènes distincts qui ne peuvent s'analyser comme un tout global. L'analyse des processus de décision de l'altermobilité, dans le registre des faits, montre que le changement modal est principalement lié à des contraintes et des opportunités avec lesquelles les personnes composent leurs habitudes de déplacements. Dans ces processus, les valeurs écologiques, entendues comme idéal d'écologie et de préservation de l'environnement, interviennent en amont et en aval du changement modal. En amont tout d'abord, sous la forme d'une réflexivité sur les pratiques, qui place les personnes dans une disposition favorable au changement. En aval ensuite, sous la forme de jus- tifications qui participent à la légitimation des nouvelles habitudes modales par l'invocation de valeurs largement admises dans la population.

Les valeurs écologiques peuvent néanmoins influencer l'ordre des faits comme c'est le cas dans les processus de changement engagés. Sous l'effet d'un processus de réduction de la dissonance cognitive, certains altermobilistes mettent en accord leurs pratiques avec leurs manières de penser en matière d'environnement. Cette action rationnelle en valeurs relève, de notre point de vue, de l'action militante. Ainsi, le lien implicite établi entre prise de conscience environnementale et changements de pratiques de déplacements nous semble largement surévalué par les acteurs qui cherchent à développer les «bonnes pratiques » en matière d'écologie au quotidien.

Les altermobilités ne nécessitent ainsi pas de valeurs écologiques pour être adoptées; de même, les valeurs écologiques ne se construisent pas nécessairement dans la pratique des altermobilités. Ainsi, les valeurs écologiques ne sont ni nécessaires, ni suffisantes à l'adoption d'altermobilités. Vouloir expliquer la «schizophrénie écologique », soit le fossé entre valeurs et pratiques écologiques, ne peut se faire sans élargir la perspective à l'ensemble des valeurs mobilisées dans l'action, mais aussi et surtout aux contraintes et opportunités avec lesquelles agissent les personnes sous peine de ne rendre compte que d'une perspective tronquée voire déterministe de la réalité sociale. L'explicitation des liens entre valeurs et pratiques en matière de mobilités durables montre, en effet, que les altermobilités ne peuvent être seulement jugées à l'aune de leur valeur écologique.

\section{Bibliographie}

Becker H.-S., [1963] 1985. Outsiders, études de sociologie de la déviance, Paris, Métailié, 249 p.

Becker H.-S., 2002. Les ficelles du métier, comment conduire sa recherche en sciences sociales, Paris, La Découverte, 352 p.

Berger P., Luckmann T., [1986] 2006. La construction sociale de la réalité, Paris, Armand Colin, 357 p.

Bonte P., Izard M., 2002. Dictionnaire de l'ethnologie et de l'anthropologie, Paris, PUF, 842 p.

Bozonnet J.-P., 2009. Environnement, nature, techniques, in Bréchon P. Tchernia J.-F. (dir.), La France à travers ses valeurs, Paris, Armand Colin, p. 122-142. 
BrÉCHON P. (dir.), 2000. Les valeurs des Français. Évolution de 1980 à 2000, Paris, Armand Colin, 280 p.

Chavanon O., Joly O., Laforgue D., Raymond R., Tabois S., 2011. Le scénario facteur 4 : les rhétoriques institutionnelles au regard des conduites ordinaires en matière de consommation d'énergie, Développement durable et territoires, Vol. 2, n 1 , mars 2011, mis en ligne le 25 février 2011 , consulté le 12 février 2013. URL: [http://developpementdurable.revues.org/8785].

Dobré M., Juan S. (dir.), 2009. Consommer autrement, La réforme écologique des modes de vie, Paris, L'Harmattan, $320 \mathrm{p}$.

Dunlap R.E., Van Liere K.D., Mertig A.G., Jones R.E., 2000. Measuring endorsement of the new ecological paradigm: A revised NEP scale, Journal of Social Issues, vol. 56, $\mathrm{n}^{\circ} 3$, p. 425-442.

Festinger L., 1957. A theory of cognitive dissonance, Stanford, Stanford University Press, 291 p.

Flamm M., 2004. Comprendre le choix modal : les déterminants des pratiques modales et des représentations individuelles des moyens de transport, thèse de doctorat, EPFL, 307 p.

Goodwin P.B., 1985. Évolution de la motivation des usagers en matière de choix modal, Table ronde CEMT, nº 68, p 65-94.

Grossetti M., 2006. Limprévisibilité dans les parcours sociaux, Cahiers Internationaux de Sociologie, $\mathrm{n}^{\circ}$ 120, Paris, PUF, p. 5-28.

Heinich N., 2006. La sociologie à l'épreuve des valeurs, Cahiers internationaux de sociologie, vol. 2, $\mathrm{n}^{\circ} 121$, p. 287-315.

Kaufmann J.-C., 1997. Le cour à l'ouvrage, théorie de l'action ménagère, Paris, Nathan, 240 p.
Kaufmann V., 2000. Mobilité quotidienne et dynamiques urbaines, la question du report modal, Lausanne, Presses polytechniques et universitaires romandes, $280 \mathrm{p}$.

Kaufmann V., Bergman M., Joye D., 2004. Motility: mobility as capital, International Journal of Urban and Regional Research, Vol. 28 n $^{\circ}$ 4, p. 745-756.

La Branche S., 2012. La schizophrénie écologique : le cas des déplacements quotidiens à Lyon, VertigO, Hors-série 11 , mai 2012, mis en ligne le 7 mai 2012, consulté le 11 mars 2013. URL : [http://vertigo.revues.org/1 1754].

Lahire B., 1998. L'homme pluriel, les ressorts de l'action, Paris, Nathan, 271 p.

Leroi-Gourhan A., 1965. Le geste et la parole, Paris, Albin Michel, $210 \mathrm{p}$.

Poitou J.-P., 1974. La dissonance cognitive, Paris, A. Colin, $127 \mathrm{p}$.

Schwartz S. H., 2006. Les valeurs de base de la personne: théorie, mesures et applications, Revue française de sociologie, vol. $47 \mathrm{n}^{\circ} 4$, p. 929-968.

VinCENT S., 2008. Les « altermobilités " : analyse sociologique d'usages de déplacements alternatifs à la voiture individuelle. Des pratiques en émergence?, thèse de doctorat en sociologie, Université Paris 5 - René Descartes, 416 p.

Vincent-Geslin S., 2010. Altermobilités, mode d'emploi, Déterminants et usages de mobilités alternatives au tout voiture, CERTU, coll. « Débats », 169 p.

Weber M., [1921] 1971. Économie et société, Paris, Plon, 650 p. 